mended to any university or research library with an interest in sound bibliographic and scholarly materials.

\title{
KONRAD EISENBICHLER
}

University of Toronto, Victoria College

Guido Pugliese, ed. Perspectives on Nineteenth-Century Italian Novels. Ottawa: Dovehouse Editions, 1989. Pp. 182.

Il volume Perspectives on Nineteenth-Century Italian Novels, a cura di Guido Pugliese, raccoglie una selezione di relazioni tenute all'omonimo convegno svoltosi presso Erindale College (University of Toronto) nell'ottobre 1986. Il convegno è stato organizzato in onore del Professor S. Bernard Chandler il quale per anni, in veste di Direttore di Dipartimento, si è prodigato per sviluppare i programmi d'Italianistica nella suddetta università. Studioso dell'Ottocento, Chandler ha dedicato ricerche assai pregevoli soprattutto a Manzoni e Verga. Sono questi, infatti, i due autori a cui il volume rivolge particolare attenzione.

Oltre una tabula gratulatoria e una breve nota biografica su Chandler seguita da un elenco delle sue pubblicazioni, apre il volume un'agile introduzione del curatore che non solo esamina singolarmente i vari contributi ma offre alcune considerazioni molto vivaci sul genere narrativo.

Il primo saggio, di Anthony Verna, affronta il problema della natura frammentaria delle Ultime lettere di Jacopo Ortis mettendo in luce i rapporti tra componenti stilistico-formali e implicazioni di ordine epistemologico. La frammentarietà narrativa è ricondotta alla caduta di un modello di totalità/unità e, quindi, ad una diversa modalità di interazione col reale. Nel romanzo del Foscolo, l'accoppiamento di narrazione epistolare, diaristica, autobiografica da una parte, e ricostruzione biografica dall'altra (quest'ultima affidata al personaggio di Lorenzo), svolge la funzione di ridurre la realtà a frammenti destinati a non ricostituirsi in una rassicurante organicità. Facendo convergere analisi formale e discorso etico-ideologico, Verna molto abilmente riesce ad illuminare l'interazione tra piano dell'espressione e piano dei contenuti consegnandoci una lettura aggionata del romanzo e attenta a non scindere i suoi molteplici elementi costitutivi.

Sei dei saggi che seguono sono dedicati a I promessi sposi. Adottando una pluralità di prospettive critiche, essi prendono in esame numerosi punti nevralgici del romanzo (il problema del realismo, gli artifici retorici, le implicazioni ideologiche, il sostrato emotivo) e riescono ad avanzare alcune proposte di lettura decisamente stimolanti e, in vari casi, dense di ramificazioni soprattutto di natura formale ed ideologica.

Gian Paolo Biasin si inoltra nel territorio poco esplorato della rappresentazione del cibo riuscendo ad aprirlo ad interessanti risvolti non solo di ordine estetico ma politico, antropologico e sociale in genere. Il motivo del nutrimento, connesso a quello della carestia e della fame-avverte Biasin-è sviluppato attraverso vari episodi che vengono a formare ricorrenti microstorie e a svolgere la funzione di veri e propri anelli narrativi. Il cibo illumina non solo rapporti sociali ed economici dei singoli personaggi, ma aiuta a dipingere anche i loro tratti psicologici. Particolarmente rilevanti le osservazioni relative alle interazioni tra il piano narrativo (necessità di ordine mimetico) e le proiezioni ideologiche di Manzoni. I riferimenti culinari, 
sostiene giustamente Biasin, diventano connotatori semantici che vanno oltre le esigenze di realismo e di mimesi (ricostruzioni ambientali ecc.). Alla rappresentazione del cibo sono infatti legate connotazioni simboliche anche di tipo religioso. Tale procedimento - qui Biasin dilata in modo molto sostanzioso il proprio discorso - sta a dimostrare che Manzoni riesce a fondere l'urgenza di una scrittura referenziale, la quale presuppone un incontro tra parola e cosa, tra segno e oggetto designato, e l'uso della dimensione metaforica del dire tramite la quale la parola viene assunta nelle sue molteplici e virtuali possibilità vicarie.

Il saggio di Robert S. Dombroski studia le strategie narrative del romanzo come finzioni dietro cui si celano le posizioni ideologiche di Manzoni. Nell'esaminare le strutture narrative de I promessi sposi come componenti inscindibili del discorso ideologico, Dombroski ci offre uno studio che mira a rispondere in modo sicuro ed equilibrato alle questioni di ordine politico che hanno costantemente diviso la critica. Manzoni, sostiene lo studioso, stabilisce un ponte tra realtà trascendentali e quelle laico-sociali spostando, di conseguenza, la storia sul piano del divino. Il risultato è un'armonizzazione delle due sfere che comunica lo sviluppo della società borghese nelle sue evoluzioni di classe. Nel suo tentativo di integrare etica cristiana e visione del mondo borghese, Manzoni-precisa Dombroski tramite nutriti riferimenti a personaggi ed episodi - è collocabile in un'area ideologicamente moderata. Con questa lettura, lucida e ponderata, si evitano pertanto quelle interpretazioni rigide e, forse, troppo settarie che hanno incondizionatamente fatto dell'autore un assoluto progressista o, soprattutto, un pericoloso reazionario.

Segue il saggio di Giuseppe Mazzotta il quale partendo dalla rappresentazione manzoniana della biblioteca di Don Ferrante vista come espressione/ricostruzione del paradigma barocco, riesce ad aprire stimolanti interpretazioni delle componenti retorico-narrative de I promessi sposi. Nella biblioteca di Don Ferrante i rapporti tra parole e cose sono venuti irrimediabilmente a spezzarsi. La pluralità infinita dei rinvii semantici crea un universo retorico in cui tutto diventa inganno, illusione. Di fronte alla parola divenuta simulacro e alla dispersione causata dalla vastità del sapere, il mondo barocco di Don Ferrante - sostiene giustamente Mazzotta - entra in un relativismo ed uno scetticismo scnza possibilità di scampo. La scrittura, con i suoi labirintici rimandi e spostamenti, si trasforma in un coacervo di segni indecifrabili frantumando così l'cpisteme che reggeva una visione ordinata e rassicurante dell'esistere. In questa lettura di Mazzotta, per molti aspetti inedita (anche se sulla biblioteca non sono mancati studi di notevole apertura critica), Manzoni rappresenta la posizione antinomica a quella di Don Ferrante in quanto cerca di esorcizzare l'ideologia barocca tentando di ristabilire i nessi tra parole e cose, tra parole e vita.

Giuliana Sanguinetti Katz sposta il discorso in direzione psicoanalitica interpretando principalmente l'agorafobia di Manzoni in relazione ai traumi infantili e alla conversione. In sintesi, l'autore riverserebbe nella sua opera il terrore provocato dall'abbandono dei suoi genitori, il mancato superamento del complesso edipico, la paura di castrazione, istinti proibiti, ansie di punizioni. I personaggi del romanzo manzoniano sarebbero pertanto delle costruzioni emotive, figure create per colmare esigenze tutte interiori. Katz fa leva, con molta destrezza, particolarmente sulle coppie antinomiche Renzo/Don Rodrigo, Lucia/Gertrude in cui Manzoni racchiuderebbe i propri conflitti proiettandovi la figura paterna e materna. Tale interpretazione è riservata anche a personaggi come Fra Cristoforo ed Egidio, e alla stessa appassionata religiosità dell'autore vista come sicurezza ricercata per compensare l'assenza dei ge- 
nitori. In questa chiave di lettura, che si richiama nelle sue premesse fondamentali agli studi di Weiss, la risoluzione finale de I promessi sposi indicherebbe un desiderio di maturità, un superamento dei dualismi e delle opposizioni emotive.

Guido Pugliese affronta il problema del linguaggio figurativo e degli artifici retorici presenti nel romanzo alla luce di alcune recenti interpretazioni critiche. Il repertorio delle immagini (vino, pane, luminosità . . . ) evidenziato da alcuni interpreti manzoniani viene da un lato ampliato e, dall'altro, proposto come fertile chiave di lettura capace di affrontare gli orizzonti complessivi dell'opera manzoniana e di sciogliere, al contempo, vari nodi interpretativi. In modo molto lucido e convincente, Pugliese cerca di dimostrare che le immagini di guerra, teatralità, labirinticità, presenti nell' Introduzione, rappresentano il discorso manzoniano sulla storia concepita come risultato delle azioni umane e affiancata al discorso morale che prende corpo nelle immagini del tempo e della morte. La contrapposizione tra ordine storico-umano e divino-spirituale si risolve a favore di quest'ultimo. Il manoscritto ritrovato viene abilmente integrato, in questa chiave di lettura, nel discorso religioso di Manzoni inserendolo così nella sua visione cristiana del mondo.

L'ultimo saggio dedicato a I promessi sposi, di Gabriele Erasmi, ricostruisce attentamente l'influenza esercitata su Manzoni dalla narrativa di Walter Scott. Appoggiandosi a vari riferimenti biografici e a documenti epistolari, oltre agli apporti della critica che si è cimentata con l'argomento in questione, egli conclude che Manzoni deve aver letto in modo abbastanza assiduo il romanziere anglosassone prima della stesura del suo capolavoro. Erasmi giustamente mette in rilievo che ne I promessi sposi sono rintracciabili personaggi ed episodi analoghi a quelli di Scott-Manzoni addirittura adopera lo stesso titolo di un suo romanzo, The Betrothed. Inoltre, anche la trasposizione in opera di un romanzo di Scott, la Lucia di Lammermoor di Donizetti, ha costituito una notevole influenza sull'opera di Manzoni. Tuttavia, avverte Erasmi, malgrado i romanzi di Scott rappresentino per il nostro romanziere una fonte sicura di ispirazione, egli si distanzia drasticamente per la sua ideologia e in particolar modo per la sua profonda visione religiosa dei destini umani.

Mirando ad abbracciare i vari itinerari del romanzo ottocentesco, il volume include anche un lavoro su Fede e bellezza di Tommaseo. Anne Urbancic, nel tentativo di dare al romanzo una veste "sperimentale", propone che la preoccupazione principale di Tommaseo non risiede tanto in problematiche contenutistiche quanto in quelle di ordine stilistico ed estetico. In contrapposizione a varie letture critiche, Urbancic insiste che l'intento di Fede e bellezza è di sperimentare con il piano dell'espressione, con la scrittura stessa. Esso è quindi lontano dagli interessi legati al romanzo storico a cui la critica, ad iniziare da Cattaneo, ha ingiustamente cercato di rapportarlo. Questa di Urbancic rappresenta una proposta di lettura che indubbiamente si apre ad interessanti implicazioni. Comunque, si tratta di una proposta che andrebbe sostanziata da precise verifiche linguistiche e stilistiche.

Tre dei saggi che seguono esplorano da diverse angolazioni la narrativa verghiana convalidando taluni giudizi, tramite nuovi apporti e verifiche, o proponendo ipotesi di lettura, sotto vari aspetti, inedite. Il primo, di Giovanni Cecchetti, esamina il problema dei rapporti tra Verga e le teorie letterarie dell'epoca, in particalare quelle avanzate dal naturalismo francese. Tramite una serie assai nutrita di riferimenti alle novelle e ai romanzi, e con l'appoggio di dichiarazioni di poetica e documentazione epistolare, Cecchetti apporta numerose e sostanziose osservazioni che aiutano a distanziare Verga dalla corrente francese e a rivendicare una sua poetica personale ed autonoma. 
La narrativa di Verga - sottolinea Cecchetti-trova la propria linfa nel mondo stesso, nella creazione di personaggi estrapolati dal reale e non in teorie letterarie già confezionate. Anzi, insiste Cccchetti, la grandezza e la modernità del romanziere siciliano risiedono nelle sue straordinarie capacità di inventare un proprio linguaggio narrativo, un proprio stile. Giustamente egli avverte che la narrativa verghiana non si basa su una semplice trascrizione del siciliano ma su una vera invenzione linguistica in lingua italiana.

Alle interpretazioni di Verga che si situano all'interno dei modelli di realismo e di mimesi, Richard Cavell oppone una lettura che tenta di allontanarsi da tali presupposti ricorrendo alle teorie decostruttive di Derrida. Facendo leva su alcune premesse derridiane (voce/scrittura, presenza/assenza, l'impossibilità di referenti non mediati dal linguaggio, lo srittore come prodotto del linguaggio), Cavell si impegna a dimostrare che un romanzo come I Malavoglia è innanzitutto un'operazione metalinguistica condotta tramite modelli di linguaggio letterario che si richiamano, tra gli altri, a Dante, Leopardi e Manzoni. Il saggio di Cavell rivela una notevole dimestichezza con le teorie decostruttive e potrebbe indubbiamente incontrare non pochi consensi. Si ha, tuttavia, l'impressione che l'impianto teorico su cui Cavell basa il proprio discorso non corrisponda in tutte le sue implicazioni all'oggetto di studio. Insomma, viene il dubbio che per la lettura che ci viene proposta forse non era necessario scomodare così tanto Derrida.

Nell'ultimo saggio dedicato a Verga, S. Bcrnard Chandler mette lucidamente in evidenza che il motivo portante della narrativa verghiana, dalla prime prove a I Malavoglia, è costituito da un desiderio dell'ignoto, da sogni di esistenze-spesso accompagnati da sentimenti d'amore - che si pongono in antitesi alla realtà effettiva dei personaggi. Tuttavia, sostiene giustamente Chandler, ne I Malavoglia scompare l'elemento amoroso e l'ignoto cessa di essere vissuto all'insegna di una magica seduzione. 'Ntoni viene a comprendere la propria realtà e l'ignoto non diventa altro che il luogo in cui risolvere $\mathrm{i}$ problemi sociali ed economici del vivere. Legato al pessimismo verghiano, l'ignoto è destinato $o$ al fallimento (in quanto sempre irraggiungibile $o$ smentito dalla dura esperienza della vita) o è posto su un piano tutto economico e sociale che viene irrimediabilmente a cancellare le connotazioni di seduzione. Con un contributo che sicuramente avvalora una certa linea della critica verghiana, Chandler conclude che la versione del mondo di Verga è tutta racchiusa in tale negatività.

I due saggi che chiudono il volume esaminano rispettivamente la narrativa di De Roberto e di Salgari. Il primo, di Mauro Buccheri, propone una rilettura in chiave utopico-nichilista de $L$ 'imperio aprendolo a numerose implicazioni estremamente significative per affrontare un'attenta sistemazione dell'opera di De Roberto. Infatti, Buccheri ci presenta un De Roberto tutto proicttato in avanti verso quei rivolgimenti estetico- epistemologici che caratterizzano due altri autori a cavallo tra Otto e Novecento, Svevo e Pirandello. L'imperio, con le sue strategie narrative e con una rinnovata versione del mondo-di cui Buccheri coglie molto attentamente soprattutto le stratificazioni apocalittiche-si allontana dal territorio veristico c si spinge da una parte verso il nulla (incorporando una lezione che va da Leopardi a Schopenhauer, a Nietzsche) e, dall'altra, verso l'utopia negativa di matrice socialista. Nelle sue tensioni e ansie esistenziali, nella sua visione di un'umanità malata e tarata alle radici, $L$ 'imperio presenta un clima di imminente catastrofe individuale e collettiva che non si può fare a meno-avverte opportunamente Buccheri-di affiancare a quello presente ne La coscienza di Zeno. 
Il saggio di Sergio M. Gilardino ricostruisce un modello dell'eroe salgariano inserendolo nell'ottica del titanismo. Superando tutti quei pregiudizi critici che relegano l'opera di Salgari esclusivamente alla lettura per l'infanzia, Gilardino riesce a riallacciare il personaggio e il mondo di Salgari alla tradizione romantica (Goethe, Büchner, Leopardi, Foscolo) e al superomismo di Nietzsche mediato dall'eroe decadente dannunziano. Gilardino riesce, quindi, molto agevolmente, a dilatare gli spazi della narrativa salgariana e a problematizzarne gli orizzonti. Secondo Gilardino, essa si discosta profondamente dalla tradizione italiana la quale, ad iniziare dal romanzo manzoniano, era tutta ripiegata entro i confini del familiare e dell'ordinario. Nella costante ricerca dei brividi dell'esotico e dell'ignoto, i romanzi di Salgari rivelano non solo l'eversiva ribellione dell'eroe romantico, ma una tensione nevrotica - esplicitata dall'esplorazione dell'onirico e delle zone recondite e minacciose dell'inconscio-avvicinabile alla sensibilità di un Poe o un Baudelaire. Un'immaginazione fertile e problematica quella di Salgari, quindi, un'immaginazione che, sebbene - precisa appropriatamente Gilardino-non sia aliena da certi esiti stucchevoli e fumettistici, riesce a creare un universo fantastico denso di connotazioni e agganci letterari.

In conclusione, Pugliese è riuscito egregiamente a consegnarci una raccolta di saggi che, nel loro insieme, non solo rappresentano un'attenta ricognizione dei percorsi e degli orientamenti presenti nella narrativa ottocentesca, ma indagano, in vari casi, sintomi e preannunci di quegli sviluppi che verranno a caratterizzare il romanzo di questo nostro Novecento.

\section{JOHN PICCHIONE}

York University

Mario Santoro e Lucia Miele. Due maestri dell'Ateneo Napoletano: Francesco Torraca e Giuseppe Toffanin. Napoli: Federico \& Ardia, 1990. Pp. 164.

In this book, the critical viewpoints of Torraca and Toffanin are examined as they developed over the years, with illustrations from their works. The relationship of the two critics to Neapolitan and southern Italian culture is also discussed, as are their attitudes and activities as professors at the University of Naples. The authors of the volume have included complete bibliographies of the critics' publications as well.

Lucia Miele notes that right from his earlier writings Torraca desired an integration of opposing methods, such as the historical and the aesthetic. He was influenced by Settembrini for the former, while De Sanctis's Saggi critici led him to regard form as a substantial element in the content of a text, a spontaneous expression of a unity of feeling. From these two writers Torraca also developed his interest in southern Italian literature as an independent project. In his pedagogical activity, he viewed artistic works as playing a central role in civilization, and with his penetrating analysis enabled them to be enjoyed by their readers. Torraca met Croce at least as early as 1885 , but their correspondence ended in 1933, following a reproof from Torraca.

Miele describes Torraca's work on Dante, Petrarch and Boccaccio, especially his criticism of Dante, and alludes to the critic's fascination with the feminine element. His knowledge of the historical and cultural pattern of the Middle Ages was vast, while he showed a lively interest in southern culture of the Renaissance. In his Rimatori napoletani del Quattrocento, he identifies two tendencies, the Petrarchan 